\title{
High field side measurements of non-thermal electron cyclotron emission on TCV plasmas with ECH and ECCD
}

\author{
P.Blanchard, S.Alberti, S.Coda, H.Weisen, P.Nikkola, \\ I.Klimanov \\ Centre de Recherches en Physique des Plasmas, \\ Association EURATOM - Confédération Suisse, \\ École Polytechnique Fédérale de Lausanne, CH-1015 Lausanne, Switzerland
}

\begin{abstract}
Measurements of electron cyclotron emission from the high field side of the TCV tokamak have been made on plasmas heated by second and third harmonic $\mathrm{X}$-mode Electron Cyclotron Heating (ECH) and Electron Cyclotron Current Drive (ECCD). Suprathermal Electron Cyclotron Emission (ECE), up to a factor of 6 in excess of thermal emission, is detected in the presence of second harmonic X-mode (X2) ECCD and of third harmonic X-mode (X3) ECH. The measured ECE spectra are modelled using a bi-Maxwellian describing the bulk and the suprathermal electron populations. Suprathermal temperatures between $10-50 \mathrm{keV}$ and densities in the range $1 \cdot 10^{17}-6 \cdot 10^{18} \mathrm{~m}^{-3}$ are obtained, and correspond to $3-15$ bulk temperatures and $1 \%-20 \%$ bulk densities. Good agreement between ECE suprathermal temperatures and energetic photon temperatures, measured by a hard X-ray camera, is found. For optically thin X3 Low Field Side (LFS) injection in presence of X2 CO-ECCD, the suprathermal population partly explains the discrepancy between global and first pass absorption measurements.
\end{abstract}

\section{Introduction}

In tokamak experiments, several operational regimes tend to create non-Maxwellian distribution functions: ion beam heating[1], dc electric fields[2], neutral beam injection[3], lower hybrid wave injection[4, 5] or ECH or ECCD injection[6, 7]. Characterisation of the non-thermal electron population in tokamak plasmas is of great importance especially in electron cyclotron heating and current drive experiments using both optically thick and optically thin harmonics of the electron cyclotron frequency $\Omega_{c e}$. The presence of such a suprathermal population strongly affects the magnitude and the spatial location of the wave absorption.

In the case of a Maxwellian plasma, the optical thickness $\tau^{X n}$ for the X-mode at the $n$-th harmonic $(n \geq 2)$ is proportional to $n_{e}\left(k_{B} T_{e} / m_{e} c^{2}\right)^{n-1}[8]$, where $n_{e}$ and $T_{e}$ are the electron density and temperature. X3 absorption is hence much weaker than X2 absorption. On TCV, in the thermal bulk, X2 is usually optically thick, while X3 
is optically thin. Injection of high power electron cyclotron waves in extraordinary polarisation mode at the second optically thick harmonic (X2) and/or third optically thin harmonic (X3) of the electron cyclotron frequency in ECH and/or ECCD injection configuration creates a non-thermal electron population, which has been shown to increase single-pass absorption for X3 Low Field Side (LFS) injection[9]. In X2-ECCD preheated plasmas, a seed suprathermal tail is generated and for LFS X3 injection, the X3 EC beam is first partly absorbed on the thermal bulk at the cold resonance and then interacts with the suprathermal population by increasing this non-thermal population and by increasing its absorption efficiency.

To study suprathermal electron populations, TCV has been equipped with an ECE radiometer working in the $\mathrm{X} 2$ frequency range and connected to a choice of two receiving antennae on the High Field Side (HFS) of the torus. This is unlike most ECE systems on other devices, which have antennae placed on the LFS of the torus[8, 10, 11]. For a LFS radiometer, the ECE from non-thermal electrons, which is relativistically frequency down-shifted, passes through the thermal electron cyclotron resonance where it is reabsorbed if the plasma is optically thick. The measured ECE is then thermal, losing the non-thermal information. In the present arrangement with antennae at the HFS, down-shifted radiation is observed without absorption at the thermal resonance.

The paper is organized as follows. In the next section, a brief description of the experimental set-up comprising the ECH/ECCD systems together with a Hard X-Ray (HXR) camera used on TCV is given. In section 3, the new ECE radiometer with HFS lines of sight is described followed by a description of the basic ECE physics. The nonthermal origin of the ECE spectrum in typical ECH/ECCD heated plasmas is discussed in subsection 4.1 while the influence of the ECH-ECCD launching geometry on the ECE spectra is examined in detail in subsection 4.2. In section 5, a simplified method used to model our experimental ECE spectra using a bi-Maxwellian distribution function for the electron population is described. The central suprathermal temperatures and densities obtained in this approximation are then discussed and compared with HXR measurements.

\section{TCV tokamak and diagnostics}

The TCV tokamak[12] is a medium-sized device with a major radius $R=0.88 \mathrm{~m}$, minor radius $a=0.25 \mathrm{~m}$ and a typical toroidal vacuum magnetic field $B_{\phi} \leq 1.54 \mathrm{~T}$. It is equipped with a flexible electron cyclotron heating and current drive system composed of six gyrotrons operating at the second harmonic $(82.7 \mathrm{GHz})$ and three gyrotrons at the third harmonic $(118 \mathrm{GHz})$ of the electron cyclotron frequency. Each gyrotron can deliver up to $0.5 \mathrm{MW}$ of microwave power. The design of the launchers, composed of movable mirrors, allows the injection of microwave power over a wide range of toroidal and poloidal angles $[9,13]$. The typical plasma cross section used in this study is shown in Fig.1. The two cold resonances for the X2 and X3 are represented in this figure and are separated by about $50 \mathrm{~mm}$ with the X3 resonance being on the LFS. 




Figure 1. Poloidal view of TCV with the antenna patterns of the two lines of sight of the radiometer and the two cold resonances of the gyrotrons X2 (vertical full line) and X3 (vertical dashed line). The two dash-dotted lines show the innermost and outermost chords of the HXR camera.

To study the suprathermal electron population, TCV is equipped with a hard X-ray camera $[14,15]$ on loan from Tore Supra. This camera measures the spatial and spectral distribution of the bremsstrahlung emission in the 10-200keV range with 14 vertical chords with energy resolution of $\sim 7 \mathrm{keV}$. The innermost and outermost chords are represented in Fig.1. In presence of a non-thermal electron population, the logarithm of the energy spectrum reveals the existence of two main components: one corresponding to the bulk and one to the non-thermal part in the high energy channels. An exponential fit to the latter part of the spectrum, which can be written as $d \gamma / d E=A e^{-E / T_{\gamma}}$, where $\gamma$ is the emissivity, provides a characterization of the suprathermal radiation by the photon temperature $T_{\gamma}$ and the normalisation factor $A$.

The electron bulk temperatures and densities are measured using a Thomson Scattering (TS) system[16] with a repetition time of 50ms. Global absorption measurements of the $\mathrm{X} 3$ beam are performed with a fast response diamagnetic loop (DML)[17] in presence of modulated X3 ECH.

\section{ECE radiometry on TCV}

In this section we present the new super-heterodyne ECE radiometer performing ECE measurements of the HFS second harmonic of $\Omega_{c e}$ in X-mode. Then a description of the two lines of sight situated on the HFS on the central column of TCV is done followed by some basic ECE physics overview. In presence of non-thermal electrons, total ECE observed by these viewing chords is a combination of bulk and non-thermal ECE and allows us to study suprathermal ECE. 


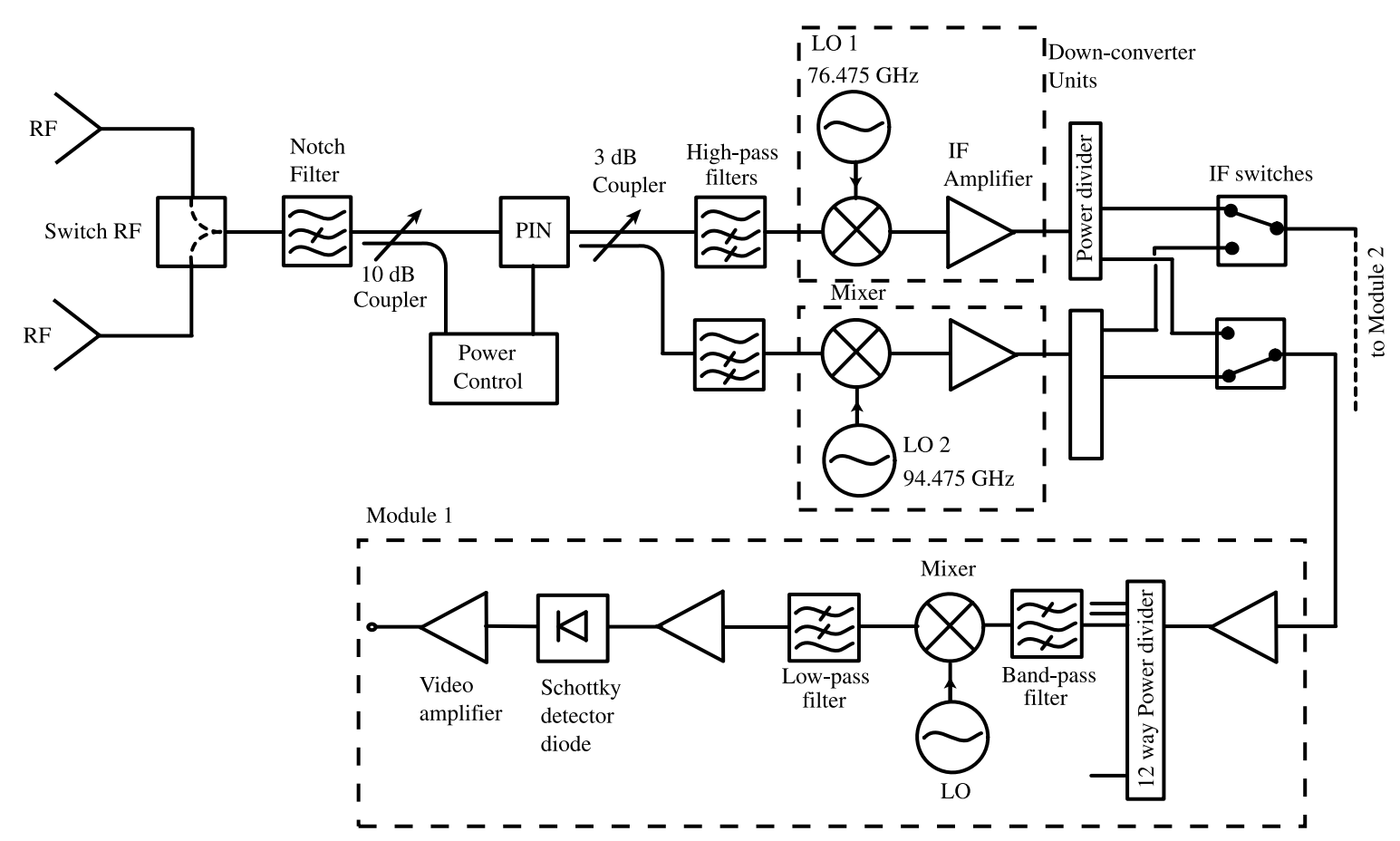

Figure 2. Schematic diagram of the super-heterodyne radiometer

\subsection{Super-heterodyne radiometer}

The 24 channels of the TCV super-heterodyne radiometer cover the frequency range $78.475-114.475 \mathrm{GHz}$, corresponding to the second harmonic of $\Omega_{c e}$ at the HFS. The frequency bandwidth of each channel is $750 \mathrm{MHz}$, corresponding to a radial resolution between $0.4 \mathrm{~cm}$ (edge) and $1 \mathrm{~cm}$ (centre) with respect to the cold resonance. It uses a two-stage frequency converter scheme as described in Fig.2. At the first stage, the ECE radiation, through the two downconverter units, is converted to a first Intermediate Frequency (IF) signal with $2-20 \mathrm{GHz}$ bandwidth through two Local Oscillators (LO) at the following frequencies: $\mathrm{LO} 1=76.475 \mathrm{GHz}$ and $\mathrm{LO} 2=94.475 \mathrm{GHz}$. The IF band is 2 to $20 \mathrm{GHz}$ for both converters, so that their down-converted frequency bands are respectively $78.475-96.475 \mathrm{GHz}$ and $96.475-114.475 \mathrm{GHz}$. Then the IF signals are sent through IF switches to two modules. Each module is composed of a 12-way power divider. Each of the 12 branches is composed of a second downconverter part, the corresponding LO being at a fixed frequency between 2 and $20 \mathrm{GHz}$. In total $2 \times 12=24$ separate LO with different frequencies are used and there is no overlap between the 24 bands. The second mixing stages are followed by a $375 \mathrm{MHz}$ low-pass filter, thus defining the channel bandwidth to be equal to twice the filter bandwidth i.e. $750 \mathrm{MHz}$. This arrangement offers the choice of either covering the entire band with a channel spacing of $1.5 \mathrm{GHz}$ or one of the two sub-bands with a channel spacing of $750 \mathrm{MHz}$. This choice is enabled by operation of the two IF switches. By means of these switches, each of the two modules can be connected to each of the downconverter units. This allows us to change both the bandwidth and the spatial resolution. At the output, consistently with 
the video bandwidth of $40 \mathrm{kHz}$ of the video amplifiers, the 24 signals are acquired at a sampling rate of $80 \mathrm{kHz}$. Two protection systems are implemented for protecting the radiometer against ECH power. The first consists of two notch filters with up to $70 \mathrm{~dB}$ signal attenuation in a bandwidth of $\pm 300 \mathrm{MHz}$ centred at the gyrotron frequencies. The width of these filters is chosen to accommodate the frequency shift of the gyrotron frequency $(0 \rightarrow 150 \mathrm{MHz}$ for $\mathrm{X} 2$ and $0 \rightarrow 300 \mathrm{MHz}$ for $\mathrm{X} 3)$ due to thermal expansion of the gyrotron cavities during the heating pulse. The second is a fast power detection system coupled to a PIN attenuator that attenuates the input power by $40 \mathrm{~dB}$ in $40 \mu \mathrm{s}$ if the input power at the $10 \mathrm{~dB}$ directional coupler exceeds $10 \mathrm{~mW}$.

\subsection{Viewing chords and RF transmission lines}

One of the main characteristics of TCV, with its highly elongated vacuum vessel, is that one can produce a large variety of plasma shapes[12]. These plasmas are not generally centred at the same height. The density cutoff, followed from the right-hand cutoff, for the second harmonic of $\Omega_{c e}$ with extraordinary polarisation occurs, in the centre of the plasma, for local electron densities larger than $n_{e}(0) \geq 4 \cdot 10^{19} \mathrm{~m}^{-3}$ while at the HFS edge the cutoff is reached for local densities higher than $8 \cdot 10^{19} \mathrm{~m}^{-3}$. For these reasons, in order to increase the accessible parameter domain, two lines of sight perpendicular to the magnetic field at $Z=0 \mathrm{~cm}$ and $Z=21 \mathrm{~cm}$ in horizontal plans have been installed. The ECE antenna at the HFS comprises an elliptical mirror followed by a vertical scalar feed horn behind the inner wall carbon tiles. Fig.1 shows the antenna pattern of these two lines on a poloidal view of TCV. The divergence angle of the antenna pattern is $\sim 8^{\circ}$. Approximately $1 \mathrm{~m}$ of fundamental rectangular wave-guide takes the collected radiation to the upper part of the vessel. The vacuum break is provided by a $200 \mu \mathrm{m}$ thick Mylar foil. TCV is also equipped with a LFS horizontal line of sight at $Z=0 \mathrm{~cm}$ with a toroidal angle of $11^{\circ}$. It is important to note that for radiometer frequencies between 94 and $114.475 \mathrm{GHz}$, there is a harmonic overlap between the second harmonic of $\Omega_{c e}$ on the HFS and the third harmonic at the LFS as shown in Fig.3. As long as the plasma is optically thick for the second harmonic in X-mode, third harmonic ECE will not be detected. On TCV this is usually the case except in the HFS edge where both the electron temperature and density are low. In this case the plasma is optically thin for the second harmonic and third harmonic emission will be seen by the HFS radiometer channels. This corresponds normally to the plasma region $\rho_{\psi} \in[0.7-1]$ where $\rho_{\psi}$ is the square root of the normalised poloidal flux.

Due to the difficulty of performing an absolute calibration of the whole ECE system (transmission lines + radiometer) because of the in-vessel location of the antennae, the signals delivered by the radiometer are cross calibrated against Thomson scattering measurements during the ohmic phase of the discharge, prior to any ECH and ECCD injection. This leads to a calibration error for optically thin areas and for channels sensitive to harmonic overlap. Signals from this optically thin area are disregarded. 


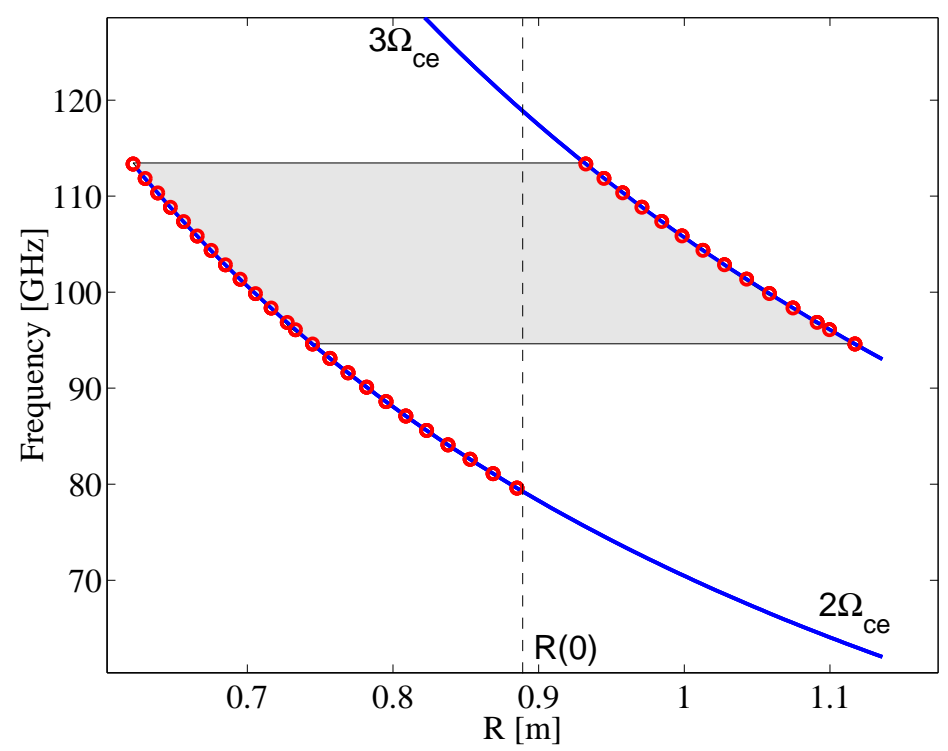

Figure 3. The second $\left(2 \Omega_{c e}\right)$ and third $\left(3 \Omega_{c e}\right)$ harmonic of the electron cyclotron frequency are drawn with the respective position of the ECE channels (circles). The vertical dashed line shows the position of the plasma centre and the grey zone shows the region of harmonic overlap

\subsection{EC emission and absorption}

For typical densities and temperatures of plasmas heated with X2 ECRH, $n_{e}(0) \approx$ $2.5 \cdot 10^{19} \mathrm{~m}^{-3}, T_{e}(0) \approx 2 \mathrm{keV}$, considering the second harmonic of $\Omega_{c e}$, the electron density is well below the density cutoff and the plasma is optically thick (the bulk optical depth $\tau_{b} \geq 10$ ): the ECE radiometer provides measurements of the electron temperature profile if the electron distribution function is Maxwellian. In this condition, the plasma behaves like a black body. The radiation temperature $T_{r}$ is defined by [18]

$$
I_{\omega}=\frac{\omega^{2}}{8 \pi^{3} c^{2}} k_{B} T_{r}\left(1-e^{-\tau_{b}}\right)
$$

where $I_{\omega}$ is the intensity of the EC radiation at the frequency $\omega$ and $\tau_{b}$ is the bulk total optical depth. For a Maxwellian plasma in thermal equilibrium $T_{r}=T_{e}$ : the radiation temperature corresponds to the electron temperature. Moreover, if the plasma is optically thick $\left(\tau_{b}>3\right), I_{\omega}$ is the black body intensity. Concerning the ECE, we will refer to the directly measurable quantity

$$
k_{B} T_{e c e}=I_{\omega} \frac{8 \pi^{3} c^{2}}{\omega^{2}}
$$

as the (apparent) ECE temperature. If the plasma is not Maxwellian, e.g. if non-thermal electrons are produced by heating the plasma with EC waves, or if the bulk is optically thin, $T_{r}$ defined by equation (1) is no longer equal to the bulk temperature.

In this study, mainly the viewing chord placed at $Z=21 \mathrm{~cm}$ has been used. With a plasma axis located at $Z=23 \mathrm{~cm}$, refraction effects are negligible since the rays are 


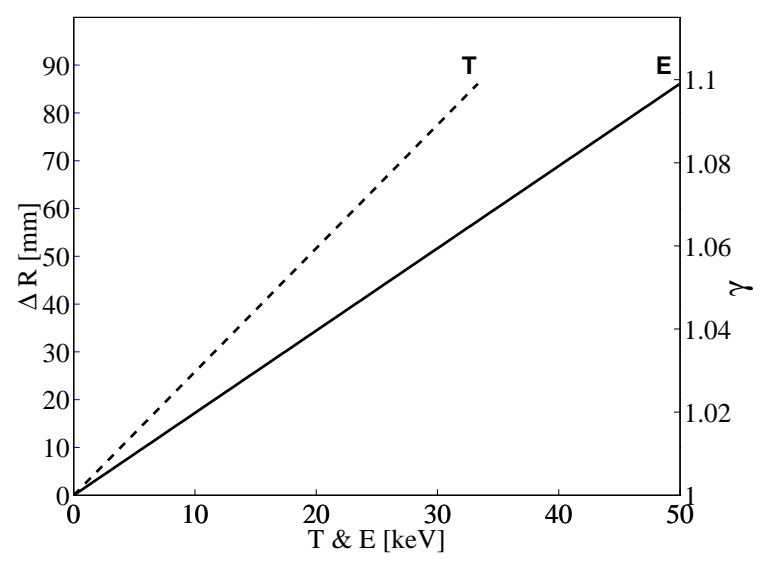

Figure 4. Correspondence for a single particle approximation between the energy (full line) or temperature (dashed line) and the corresponding radial shift (left Yaxis) or relativistic factor $\gamma$ (right Y-axis). We used the following correspondence: $E=m_{e} c^{2}(\Delta R / R(0))=m_{e} c^{2}(\gamma-1)$ and $E=(3 / 2) T$

practically parallel to the index of refraction gradient. With ECE-HFS geometry and with the target plasmas used in this study $\left(\tau_{b}(0)>10\right.$ for the second harmonic of $\left.\Omega_{c e}\right)$, we neglect wall reflections since the $\mathrm{X} 2$ resonance layer of the bulk acts as an ideal absorber.

The ECE from suprathermals with kinetic energy $E_{k}$ is frequency-downshifted by the relativistic factor $\gamma$ according to the formula $E_{k}=m_{e} c^{2}(\gamma-1)$. This corresponds to a frequency downshift $\Delta \omega=n \Omega_{c e}(\gamma-1)$ which corresponds, with respect to the cold resonance, to an apparent radial shift towards the LFS given by $\Delta R=R_{c}(1-\gamma)$ where $R_{c}$ is the position of the cold plasma resonance for the considered frequency. As one can see in Fig.4, for $E_{k}=10 \mathrm{keV}, \gamma=1.02$ which corresponds to a radial shift of about $17 \mathrm{~mm}$ for a central emission. If the radiation is observed from the HFS, this downshifted radiation propagating towards the HFS does not pass through the EC cold resonance and is unaffected by the bulk absorption. At any given observation frequency, the intensity of the EC radiation is then the result of a sequence of emissions and absorptions by electrons of different energies, occurring in different plasma regions. The radiometer channel spacing of $750 \mathrm{MHz}$ corresponds to an energy increment of $\sim 4.8 \mathrm{keV}$.

\section{HFS ECE measurements on TCV with X3 ECH in presence of X2 ECCD}

ECE measurements from the HFS of the TCV tokamak have been achieved on plasmas with X2 ECCD and optically thin X3 ECH injections. Both injections generate suprathermal electrons. In this section, we first show, in such scenarios, the nonthermal nature of the experimental HFS ECE spectra. We then present a study of the dependence of the ECE spectra on the X2 toroidal launching angle. 


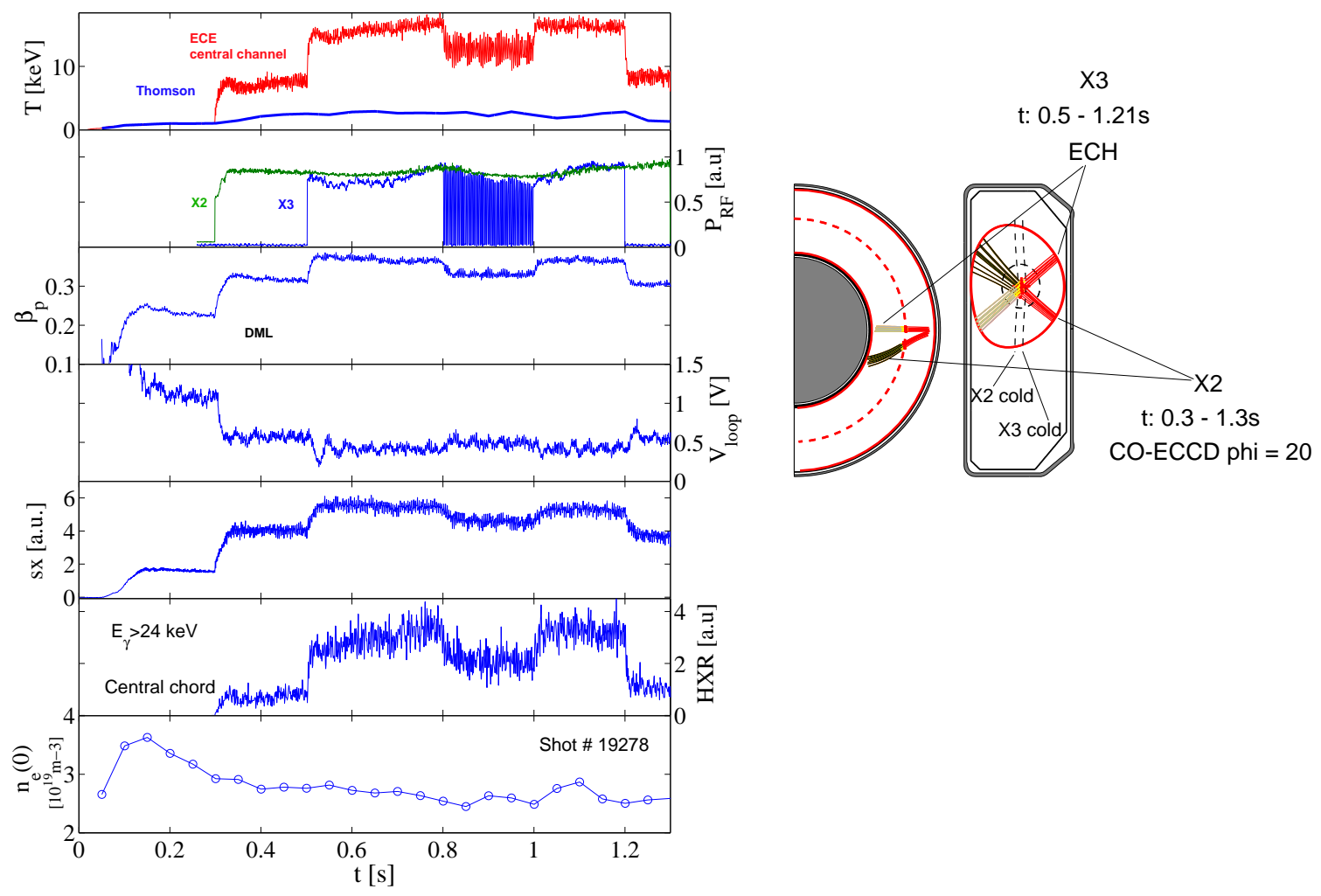

Figure 5. Left: Temporal evolution of different plasma parameters for the EC heated plasma \#19278 with $480 \mathrm{~kW}$ central X2 CO-ECCD $+20^{\circ}$ and $450 \mathrm{~kW}$ central X3 ECH. From top to bottom: 1) Central channel of the ECE (@ $\left.\rho_{\psi}=0.1\right)$ and central Thomson temperatures $\left.T_{T S}, 2\right) \mathrm{RF}$ power for $\mathrm{X} 2$ and $\mathrm{X} 3 \mathrm{ECH}$ (without and with power modulation), 3) Poloidal beta, 4) $V_{\text {loop }}$, 5) Soft x-ray signal (central chord), 6) Hard x-ray signal (central chord) with photon energies $\left.E_{\gamma}>24 \mathrm{keV}, 7\right)$ Central electron density obtained by Thomson scattering. Right: Launching geometry for the X2 and X3 beams

\subsection{Experimental evidence of the non-thermal nature of the ECE measurements}

The target plasmas used in these experiments[9] have the following parameters: $R_{0}=$ $0.89 \mathrm{~m}, a=0.24 \mathrm{~m}$, elongation $\kappa=1.31$, triangularity $\delta=0.15, B_{\phi}=1.42 \mathrm{~T}$, $n_{e}(0)=2.5 \cdot 10^{19} \mathrm{~m}^{-3}, I_{p}=200 \mathrm{kA}$. All the discharges have an ohmic phase of about $0.3 \mathrm{~s}$ followed by a X2 'preheating' phase with constant power $\left(P_{X 2}=0.45 \mathrm{MW}\right)$ from $0.3 \mathrm{~s}$ to $1.3 \mathrm{~s}$. The X3 power is applied from $0.5 \mathrm{~s}$ to $1.21 \mathrm{~s}$. The X3 power $\left(P_{X 3}=0.45 \mathrm{MW}\right)$ and the launching angle (no current drive component) are kept constant, whereas the toroidal and poloidal launching angles of the X2 ECH/ECCD are varied. As shown in Fig.3, the X2 and X3 cold resonances are separated spatially by about 50mm. They are nearly symmetric with respect to the plasma axis, the X3 resonance being on the low field side.

The typical temporal evolution of different plasma parameters during EC heated plasmas with combined X2 ECCD and X3 ECH is shown in Fig.5. In the upper part of the 
plot, which represents the most central ECE channel, one clearly sees the effect of the EC heating. X2 power is injected in CO-ECCD (toroidal launching angle $+20^{\circ}$ ) whereas X3 is in central ECH mode. With X2-ECCD only (from 0.3 to $0.5 \mathrm{~s}$ ) the ECE temperature exceeds the electron temperature by a factor of three. This EC emission is non-thermal, demonstrating, as will be discussed later, that X2 ECCD creates suprathermal electrons, confirming previous results[19]. The addition of X3 ECH leads to an increase of $T_{\text {ece }}$ by a factor of two. The X3 heating phase starts at $0.5 \mathrm{~s}$ and features a period of $100 \%$ power modulation $(0.8-1$ s) at $237 \mathrm{~Hz}$, during which a global absorption measurement is obtained with DML measurements: in this discharge $60 \%$ of the X3 ECH power is absorbed by the plasma. Single pass absorption, by an isotropic Maxwellian distribution function with $T_{b}=T_{T S}$, calculated with the linear ray tracing code TORAY-GA[20, 21, 22, 23], can only account for $30 \%$ of X3 power absorption. The difference between the DML measurements and TORAY-GA calculations indicates that half of the X3 power is absorbed mainly by the suprathermals generated by X2 ECCD. The effect of multiple reflections of the X3 beam on the inner walls of the torus on X3 beam absorption has already been investigated[17] and is negligible for X-mode central deposition. Moreover, X3 increases the suprathermal emission, demonstrating that the X3 ECH beam interacts with the suprathermal population and enhances this population. This interpretation is corroborated by results from the hard X-ray camera[24], which show a strong increase of suprathermal X-ray emission with combined X2 ECCD and X3 ECH, while the soft X-ray emission only increases moderately (Fig.5). The non-thermal origin of the ECE spectra in the presence of EC heating is also clearly demonstrated by the sawtooth activity on the ECE radiometer signals. Comparison of the spatial location of the sawtooth inversion radius obtained with the ECE radiometer signals and with soft X-ray measurements clearly shows the effect of the relativistic downshift of the ECE signals emitted by non-thermal population as illustrated in Fig.6. For a radial shift $\Delta R$, where $R$ is the spatial location of the non-shifted event, we obtain the characteristic energy of the non-thermal electrons by $E=m_{e} c^{2}(\Delta R / R)$. Based on this relation, for the typical heated plasma presented in this article, the energy estimation of such non-thermal electrons in the $\mathrm{X} 2+\mathrm{X} 3$ phase is between 30 and $45 \mathrm{keV}$ while in the $\mathrm{X} 2$ phase alone, the energy is between 15 and $35 \mathrm{keV}$.

\subsection{Dependence of the HFS ECE on the toroidal X2 launcher geometry}

In TCV, non-thermal electron cyclotron emission is generated when high power electron cyclotron waves are injected with X3 ECH and/or with X2, except with central $\mathrm{X} 2 \mathrm{ECH}$ injection (no current drive component). The dependence of the ECE on the launcher geometry has been studied on discharges with central X3 ECH and X2 central moving from shot to shot its toroidal injection angle in order to have local toroidal angle $\phi_{a b s} \in\left[-45^{\circ}-+50^{\circ}\right]$, with $\sin \left(\phi_{a b s}\right)=\mathbf{k} \cdot \mathbf{B} /|\mathbf{k}| \cdot|\mathbf{B}|$.

The sensitivity of the ECE to the X2 toroidal injection angle of the EC power is shown in Fig.7. On this figure, considering the $\mathrm{X} 2$ phase alone, we first see that if the $\mathrm{X} 2$ power 




Figure 6. Temporal evolution of ECE signals (left) and soft X-ray measurements (right) of shot \#21668. The y-axis represents the radial position of these emissions; in the ECE case, this is calculated considering the cold resonance. The amplitude of the signals is arbitrary. The horizontal grey lines shows the position of the inversion radii for both diagnostics

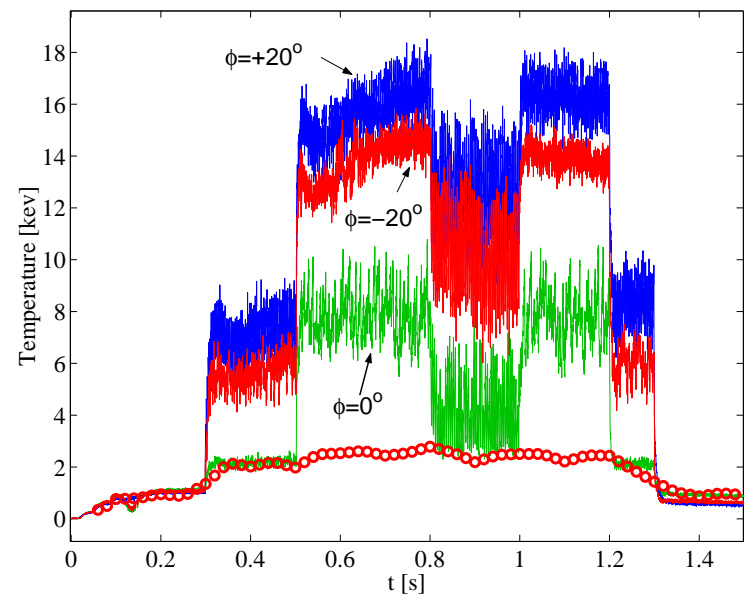

Figure 7. Temporal evolution of central $T_{e c e}$ and $T_{T S}$ (circle). The three ECE signals correspond to the central channel of the ECE for three different central X2 launching configurations with toroidal injection angle of $-20^{\circ}, 0^{\circ}$ and $+20^{\circ}$ on the same plasma target. $500 \mathrm{~kW}$ of X2 power were launched from 0.3 to $1.3 \mathrm{~s}$ and $500 \mathrm{~kW}$ of central X3 power were launched from 0.5 to $1.2 \mathrm{~s}$ with on/off modulation from 0.8 to $1 \mathrm{~s}$

is launched with zero toroidal angle, the emission is thermal: $T_{e c e}$ and $T_{T S}$ are equal and there is no evidence of a suprathermal electron population. On the other hand, if the X2 launchers have a non-zero toroidal angle, $T_{\text {ece }}$ is no longer thermal, indicating that in this configuration suprathermal electrons are generated by X2 ECCD. Moreover, we observe an asymmetry on the ECE signal between CO-ECCD and CNT-ECCD.

When central X3 ECH power is added, this asymmetry in the ECE signals with respect to the sign of the toroidal injection angle is preserved. This CO-CNT asymmetry is 

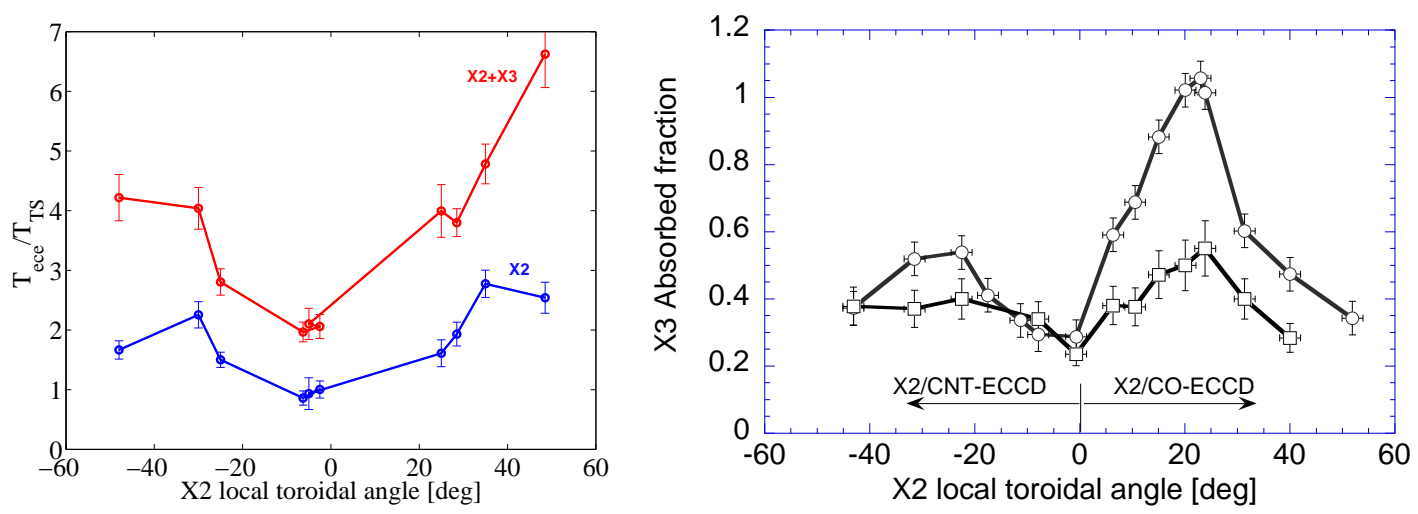

Figure 8. Left: Central temperature comparison versus the local toroidal angle for X2. The lower line is the ratio of $T_{\text {ece }} / T_{T S} @ \rho_{\psi}=0.1$ during the X2 heating phase of the shot. The upper line is the same ration during the X2+X3 heating phase. Right: Measured X3 power absorption with DML (circles) and calculated absorption with TORAY-GA (squares) versus X2 local toroidal angle

also observed in the absorption as measured with the DML $[9,17]$ and as calculated by TORAY-GA, as shown in Fig.8(right). 100\% X3 absorption has been measured with an $\mathrm{X} 2$ local toroidal angle of $+25^{\circ}$ whereas we obtain only $55 \%$ with a symmetric X2 local toroidal angle of $-25^{\circ}$. With X2 ECH preheating (injection angle $=0^{\circ}$ ), the X3 absorption is lowest with only $30 \%$ absorption. Considering the X3 absorption on the bulk with $\mathrm{X} 2 \mathrm{CO}-\mathrm{ECCD} 25^{\circ}$, we calculate that $\tau^{X 3}=0.8$ indicating that only $\left(1-e^{-\tau^{X 3}}\right)=55 \%$ of the X3 power is absorbed on the bulk. The rest being absorbed by the suprathermal population. Finally the ECE data for $\phi_{a b s}=0$ show that X3 ECH alone creates a measurable suprathermal electron population.

The global dependence of the ECE on the X2 toroidal launching angle is shown in Fig.8(left). Typical bulk temperatures in this shot-to-shot scan are $2 \mathrm{keV}$ with $\mathrm{X} 2$ only and $2.5 \mathrm{keV}$ with combined $\mathrm{X} 2 / \mathrm{X} 3$ heating. The ECE temperature is highest for $\mathrm{X} 2$ injection angles of $\pm 25^{\circ}$ with X2 heating only. During the combined heating phase the radiation temperature increases with the injection angle up to at least $+45^{\circ}$. These observations are qualitatively consistent with hard X-ray measurements, which show the same behaviour on the hard X emissivity with emissivity peaks at $\pm 25^{\circ}$ launching angle[24].

\section{ECE analysis based on a bi-Maxwellian model}

The calculation of the ECE spectra in the experiments presented in this paper would require the calculation of the electron distribution function with a Fokker-Plank code and, subsequently, using this result in an ECE code coupled to a ray tracing code. At present, the combined $\mathrm{X} 2+\mathrm{X} 3$ experiments cannot be simulated by our Fokker-Plank code CQL3D[25] because it is not yet capable of taking into account the X3 wave. 


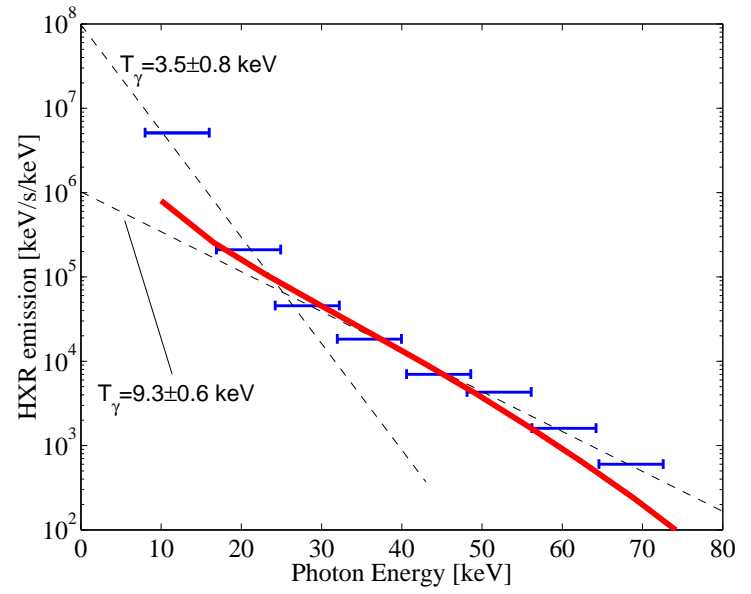

(a)

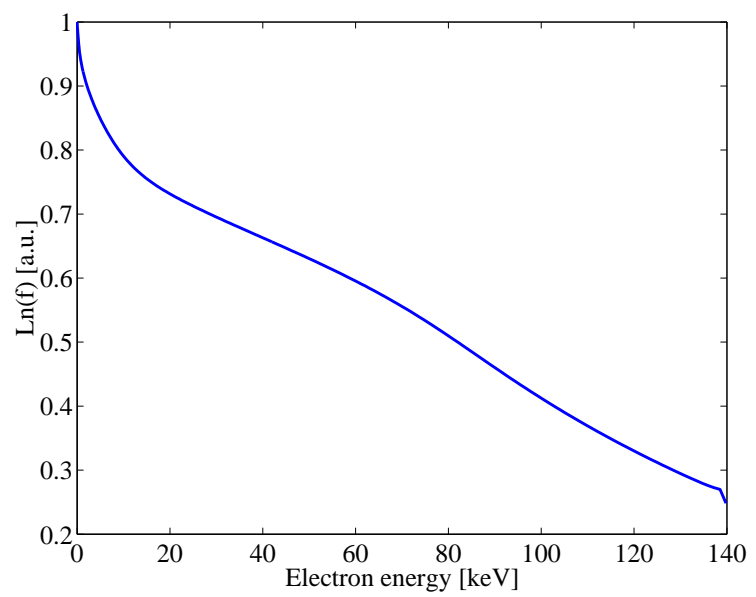

(b)

Figure 9. Results from shot \#21739 with $1.35 \mathrm{MW} \mathrm{X2}$ central injection with a $+15^{\circ}$ toroidal injection angle. (a): Comparison between central HXR experimental spectra (horizontal segments) and simulated HXR spectra (-) obtained with the CQL3D code. The two dashed lines are exponential fits to the experimental data characterizing bulk and suprathermal populations whose slopes give the indicated temperatures. By comparison, the bulk temperature measured by Thomson scattering is $3.5 \mathrm{keV}$. (b): Electron distribution function calculated with the CQL3D code integrated over the pitch-angle.

Moreover, our ECE code is at present being upgraded in order to simulate, in the future, ECE spectra with numerical distribution functions. Nevertheless, recent simulations of the experimental HXR emission spectra, obtained on plasmas with high power density X2 injections, indicates that to match CQL3D calculations with HXR experimental spectra one has to consider radial diffusion of the fast electrons with a radial diffusion coefficient larger than $D=1.5 \mathrm{~m}^{2} / \mathrm{s}[25,26]$. As shown on Fig.9(a), the logarithm of the experimental and the calculated energy spectrum of the bremsstrahlung emission clearly reveal two main components characterising the bulk, whose slope is consistent with the electron bulk temperature, and the non-thermal population, justifying a biMaxwellian approach to simulate ECE experimental spectra. In addition, as shown in reference [26], the equilibrium radial profile of the measured photon temperature can be considered as constant. This result suggests that, by including radial diffusion, the suprathermal electron temperature profile can be considered as constant.

\subsection{Description of the bi-Maxwellian simulation method}

To describe non-thermal plasmas we consider a bi-Maxwellian distribution function $f$ for the electrons: $f=(1-\eta) f_{b}+\eta f_{s}$. Thus $f$ is a linear combination of two Maxwellians, the first one, $f_{b}$, representing the bulk electrons with temperature $T_{b}$ and density $n_{b}$, 
and the second one, $f_{s}$, the suprathermal electrons with temperature $T_{s}$ and density $n_{s}$; here $n_{s}=n_{b} \cdot \eta /(1-\eta)$ and $\int f_{b} d^{3} v=\int f_{s} d^{3} v=1$ and

$$
f_{j}(r, \mathbf{v})=\left(\frac{m_{e}}{2 \pi k_{B} T_{j}}\right)^{3 / 2} \exp \left(-\frac{m_{e} v^{2}}{2 k_{B} T_{j}}\right) \quad ; \quad j=b, s
$$

No anisotropy in the distribution function is considered here; this is justified since for the heated plasmas considered in this paper, the pitch angle scattering time is of the order of 200-300 $\mu s[17]$ and much shorter than the other characteristic times determining the suprathermal dynamics. Under this assumption and for a HFS view, an expression for the local intensity is obtained as[27, 28]:

$$
I_{\omega}=\frac{\omega^{2}}{8 \pi^{3} c^{2}}\left[T_{b}\left(1-e^{-\tau_{b}}\right) e^{-\tau_{s}}+T_{s}\left(1-e^{-\tau_{s}}\right)\right]
$$

$\tau_{b}$ and $\tau_{s}$ being the respective optical depths for the second harmonic of $\Omega_{c e}$ in X-mode. For a LFS view, the corresponding equation is

$$
I_{\omega}=\frac{\omega^{2}}{8 \pi^{3} c^{2}}\left[T_{b}\left(1-e^{-\tau_{b}}\right)+T_{s}\left(1-e^{-\tau_{s}}\right) e^{-\tau_{b}}\right]
$$

In our experiments, $\tau_{b} \geq 10$ and the interest of using HFS view to observe ECE and study suprathermal electron populations is obvious. Since in our plasmas the central bulk density $n_{b}(0) \approx 2.5 \cdot 10^{19} \mathrm{~m}^{-3}$ is not much lower than the cutoff density $n_{c u t}^{X 2}(0) \approx 4.1 \cdot 10^{19} \mathrm{~m}^{-3}$, we have to take into account finite density effects to calculate the optical depths. Moreover, we have considered corrections to the optical depth calculation to take into account intermediate temperature effects, typically up to $20 \mathrm{keV}$. For a Maxwellian or bi-Maxwellian plasma with $1 / R$ space dependent magnetic field with perpendicular propagation, with reference to Bornatici et al [8], we calculate the optical depths $\tau$ as described in detail in the Appendix. For the plasmas studied in this paper, we have $n_{b}(0) \approx 2.5 \cdot 10^{19} \mathrm{~m}^{-3}$ and $T_{b}(0) \approx 2 \rightarrow 3 \mathrm{keV}$ defining a resonance region (width of the absorption coefficient at midheight) of about $0.5 \rightarrow 1 \mathrm{~cm}$ while for the suprathermals we have $n_{s}(0) \approx 0.5 \cdot 10^{17} \rightarrow 6 \cdot 10^{18} \mathrm{~m}^{-3}$ and $T_{s}(0) \approx 10 \rightarrow 50 \mathrm{keV}$ defining a resonance region of about $5 \rightarrow 8 \mathrm{~cm}$ (see also Fig.14(a) at the end of this paper). In these conditions, the maxima of the absorption coefficients for the bulk and for the suprathermal are separated by about $3.5 \mathrm{~cm}$. The ECE from the two populations then originates in two spatially separated regions of the plasma.

In the bi-Maxwellian model, if $\exp \left(-\tau_{s}\right)<<1$, the plasma radiates like a blackbody at the suprathermal temperature. As we can see in the $\tau_{s}(0)$ contour plot in Fig.10, the plasma is optically thick for central suprathermal densities higher than $3 \cdot 10^{18} \mathrm{~m}^{-3}$ and temperatures larger than $15 \mathrm{keV}$. The ECE spectra will generally be a combination of bulk and suprathermal emission from different locations, therefore, the radiation temperature cannot be related either to the bulk temperature or to the suprathermal temperature. 


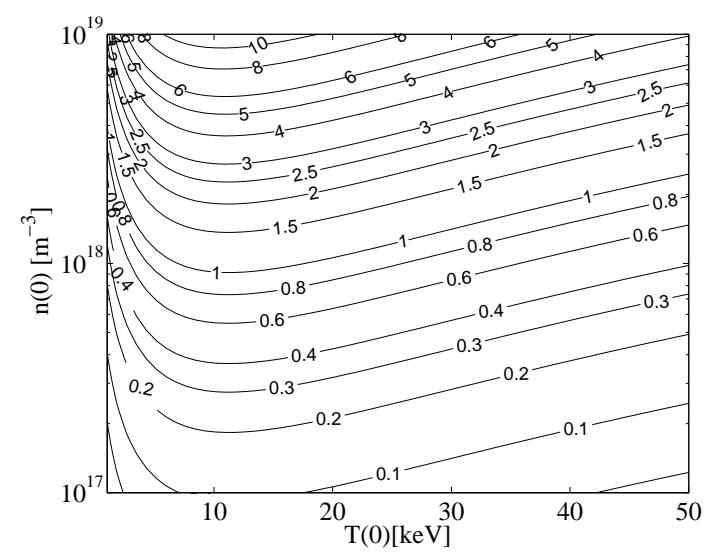

Figure 10. Contour plot of the values of the optical thickness $\tau_{s}(0)$ for the second harmonic X-mode as defined in the Appendix. The temperature and density profiles considered for this calculation are $T(r)=T(0) \cdot\left(1-(r / a)^{2}\right)^{0.5}$ and $n(r)=n(0)(1-$ $\left.(r / a)^{2}\right)^{8}$

\subsection{Application of the bi-Maxwellian model to a combined X2-X3 experiment}

We will apply a bi-Maxwellian approximation in order to estimate suprathermal temperatures and densities, taking into account relativistic effects for both bulk and suprathermal populations. Bulk and suprathermal populations have independent Maxwellian electron distribution functions defined by the spatial profiles of their respective temperatures $T_{b}(r), T_{s}(r)$ and densities $n_{b}(r), n_{s}(r)$. The bulk temperature and density profiles are obtained from Thomson scattering measurements. HXR emissivity and temperature measurements are used in order to obtain an estimate of the shape of the spatial distributions of the suprathermal density and temperature, with their central values $T_{s}(0)$ and $n_{s}(0)$ left as the two free parameters. The spatial distribution of the local HXR emissivity is reconstructed by assuming poloidal homogeneity and performing profile inversions with the Fisher regularisation method[24]. If the bremsstrahlung emissivity has an approximately exponential energy distribution, the suprathermal density is roughly proportional to the emissivity divided by $n_{e}(r) \cdot Z_{e f f}(r)$, with correction factors dependent on the photon temperature. The latter displays little spatial variation, and within the limited accuracy of our $Z_{\text {eff }}$ measurements, we can estimate the quantity $n_{e}(r) \cdot Z_{\text {eff }}(r)$ to be typically rather flat in the region of interest as well. For the purpose of the present analysis, we thus take the shape of the emissivity profile to be representative of that of the suprathermal density. The spatial distribution of the temperature of the high energy photon emission is not far from flat, whereas the emissivity profile is more peaked $[24,26]$. By fitting parabolic profiles to the HXR results, one obtains, for the shots considered in this section, that $T_{s}(r)=T_{s}(0) \cdot\left(1-(r / a)^{2}\right)^{\alpha}, \alpha \in[-0.5,0.5]$ and $n_{s}(r)=n_{s}(0) \cdot\left(1-(r / a)^{2}\right)^{\beta}, \beta \in[6,10]$, where $a$ is the minor radius of the plasma. Once we have chosen the spatial shape of the suprathermal profiles, it is possible, using equation (4), to find values of the suprathermal central density $n_{s}(0)$ and temperature $T_{s}(0)$ that satisfy, at any given 
emission frequency, the relation (equations (2) \& (4)):

$$
T_{\text {ece }}=T_{b}\left(1-e^{-\tau_{b}}\right) e^{-\tau_{s}}+T_{s}\left(1-e^{-\tau_{s}}\right)
$$

As an example, we consider the discharge \#21732 preheated with $0.45 \mathrm{MW}$ X2 power with a $+15^{\circ}$ toroidal injection angle and heated with $0.45 \mathrm{MW}$ X3 ECH power. The temperature and density profiles for the bulk are shown on Fig.11(a) and the considered shapes for the non-thermal population are shown on Fig.11(b). Experimentally it is observed that for shot \#21732 during the X2+X3 heating phase $T_{\text {ece }}=4.3 \mathrm{keV}$ on the ECE channel of $87.1 \mathrm{GHz}$. The corresponding curve in Fig.11(c) defines all the $\left[n_{s}(0), T_{s}(0)\right]$ pairs for which the bi-Maxwellian simulation gives $4.3 \mathrm{keV}$ at $87.1 \mathrm{GHz}$. Taking into account several points on the experimental ECE spectrum, it is possible, for each point, to obtain such curves as represented in Fig.11(c) for three values of $T_{\text {ece }}$. Empirically, we find that each pair of these curves always intersects and that the location of their intersection points occupies a rather narrow parameter region. We then define the average of these intersection points as the $\left[n_{s}(0), T_{s}(0)\right]$ pair for this shot while the standard deviation is used as error bar. On Fig.11(d) we can see the calculated profile for this case. The dependence of the simulated profiles on the peaking factors $\alpha$ and $\beta$ is shown on Fig.12. The peaking factor of the suprathermal temperature profile mainly affects the LFS of the simulated profiles, while that of the suprathermal density profile affects weakly the shape of the whole simulated profile.

\subsection{Suprathermal population dependence on X2-ECCD preheating power}

To study the influence of the EC power on the ECE spectra we carried out, on the same plasmas as discussed before, a shot-to-shot X2 central power scan from 0 to $1.35 \mathrm{MW}$ with $+15^{\circ}$ toroidal launcher injection angle $\left(\phi_{a b s}=+12^{\circ}\right)$ before $0.45 \mathrm{MW} \mathrm{X3}$ central $\mathrm{ECH}$ injection. Results of the bi-Maxwellian approximation applied to the X2 preheating phase of the shot are shown in Fig.13(a). $T_{s}(0)$ is approximately $7 \mathrm{keV}$ and does not depend significantly on the X2 power while $n_{s}(0)$ increases linearly from $1 \cdot 10^{17} \mathrm{~m}^{-3}$ with $P_{X 2}=0.25 \mathrm{MW}$ to $2 \cdot 10^{18} \mathrm{~m}^{-3}$ with $P_{X 2}=1.35 \mathrm{MW}$; by contrast $n_{b}(0) \approx 2.5 \cdot 10^{19} \mathrm{~m}^{-3}$ for all shots. Results of the bi-Maxwellian approximation method during the $\mathrm{X} 2+\mathrm{X} 3$ phase of the shots are shown in Fig.13(b). As the X2 power is increased from 0 to $1.35 \mathrm{MW}, n_{s}(0)$ increases linearly from $1.2 \cdot 10^{18} \mathrm{~m}^{-3}$ to $5.4 \cdot 10^{18} \mathrm{~m}^{-3}$; at the same time $T_{s}(0)$ decreases from $51 \mathrm{keV}$ to $12 \mathrm{keV}$. Moreover, $T_{s}(0)$ is nearly constant for $P_{X 2}>0.45$ MW. The suprathermal temperature in the $\mathrm{X} 2+\mathrm{X} 3$ phase is in fair agreement with the photon temperature from the HXR camera. We also see that, as the X2 power increases from 0 to $1.35 \mathrm{MW}$ with constant X3 power, the central suprathermal optical depth $\tau_{s}(0)$ increases from 0.5 to 6.1 : the suprathermal population becomes more and more optically thick. The fraction of the electron energy carried by the suprathermals, i.e. $\int n_{s} T_{s} /\left(n_{s} T_{s}+n_{b} T_{b}\right) d V$, is $15 \%$ with $0.15 \mathrm{MW}$ X2 and increases up to $40 \%$ with $1.35 \mathrm{MW}$ $\mathrm{X} 2$ power.

It is important to note that for all these shots the bulk is optically thick for $2 \Omega_{c e}$; hence 




(a)

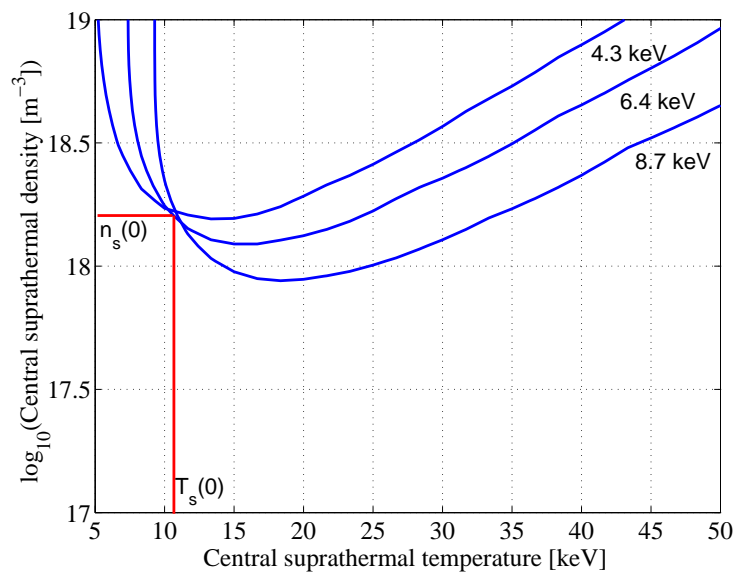

(c)

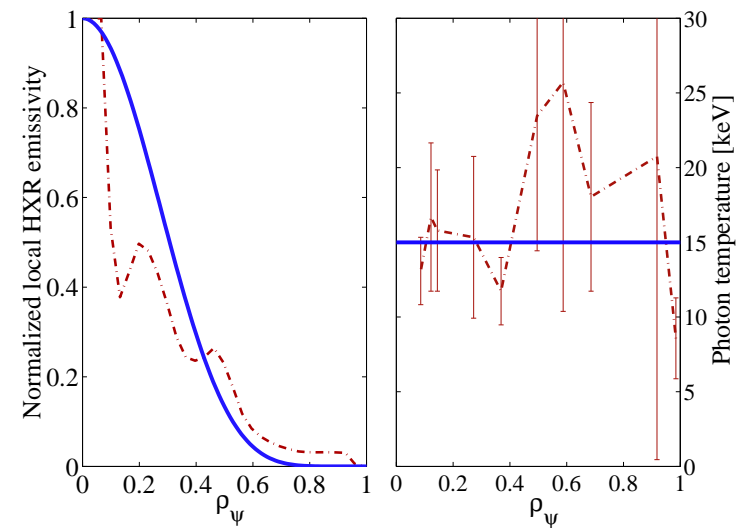

(b)

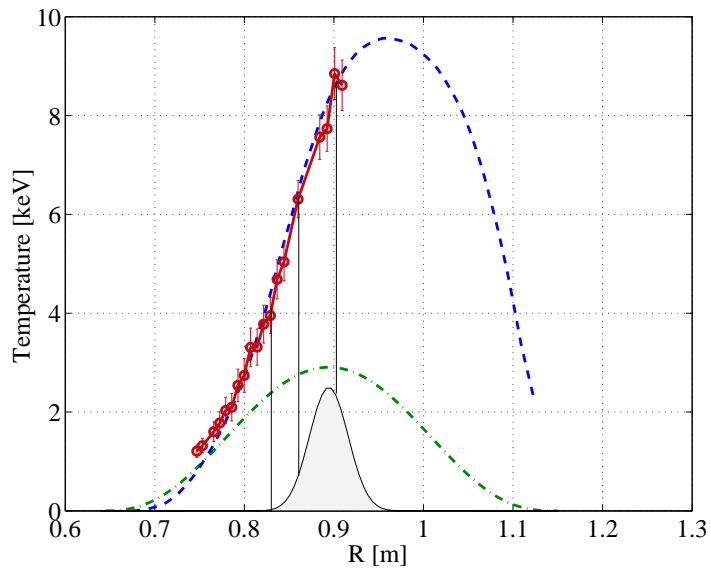

(d)

Figure 11. Results from shot \#21732 preheated with $0.45 \mathrm{MW}$ X2 power with a $+15^{\circ}$ toroidal injection angle and heated with $0.45 \mathrm{MW} \mathrm{X3} \mathrm{ECH} \mathrm{power.} \mathrm{(a):} \mathrm{Density} \mathrm{(left)}$ and temperature (right) Thomson profiles for the bulk. (b): The dash-dotted lines represent the normalised local HXR emissivity (left) and photon temperature (right) for the suprathermal population. The solid lines are the shape of the suprathermal density (left) and temperature (right) considered for the simulation. (c): Equi-temperature lines for the three values of $T_{\text {ece }}$ represented on the graph on the right. The intersection of these curves defines $n_{s}(0), T_{s}(0)$. (d): The dashed curve shows the ECE spectrum calculated with the bi-Maxwellian approximation with $n_{s}(0), \beta=8, T_{s}(0), \alpha=0$, superposed on the experimental results (circles). The dash-dotted curve shows the bulk temperature profile while the filled profile shows the EC power deposition location (arbitrary units). 

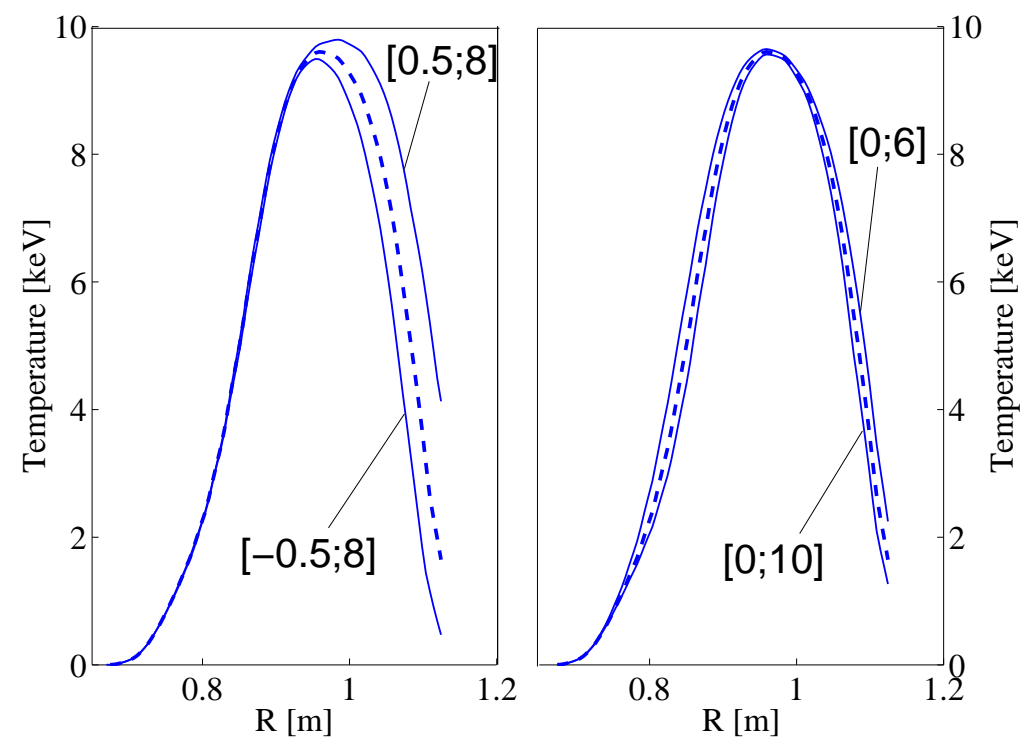

Figure 12. ECE simulated spectrum for different $[\alpha ; \beta]$ values. The dashed curves replicate the dashed curve in Fig.11(d) obtained with $[0,8]$

Table 1. Summary of the $\mathrm{X} 2$ power scan during the $\mathrm{X} 2+\mathrm{X} 3$ phase of the shots

\begin{tabular}{|c|c|c|c|c|c|c|c|}
\hline $\begin{array}{c}\text { X2 Power } \\
{[\mathrm{MW}]}\end{array}$ & $\begin{array}{c}T_{\text {ece }}(0) \\
{[\mathrm{keV}]}\end{array}$ & $\begin{array}{c}T_{T S}(0) \\
{[\mathrm{keV}]}\end{array}$ & $\begin{array}{c}T_{s}(0) \\
{[\mathrm{keV}]}\end{array}$ & $\begin{array}{c}n_{s}(0) / n_{b}(0) \\
{[\%]}\end{array}$ & $\tau_{s}(0)$ & $1-e^{-\tau_{s}(0)}$ & $\tau_{b}(0)$ \\
\hline 0 & 2.8 & 2 & 51 & 8 & 0.5 & 0.4 & 6.8 \\
0.5 & 9 & 2.8 & 12.6 & 9 & 1.4 & 0.75 & 12.6 \\
1.5 & 12 & 2.7 & 12 & 24 & 6.1 & 1 & 14 \\
\hline
\end{tabular}

equation (6) reduces to $T_{\text {ece }}=T_{b} e^{-\tau_{s}}+T_{s}\left(1-e^{-\tau_{s}}\right)$ : it is mainly the value of $\tau_{s}$ i.e. the optical thickness of the suprathermal electron population that governs the ECE. Table 1 gives an overview of these results for three different X2 power values. Without X2 power, due to the low optical depth of the suprathermal population, the ECE is nearly thermal and tends to represent the bulk temperature. Note that in this case, the effective $\tau_{s}$ to be used in equation (6) for the central channel of the radiometer is in fact equal to 0.018 and thus considerably lower than $\tau_{s}=0.5$, owing to a $10 \mathrm{~cm}$ relativistic shift towards the HFS. With $0.45 \mathrm{MW}$ of X2 power, the suprathermal population is thicker and with reference to equation (4), $T_{\text {ece }}$ is intermediate between bulk and suprathermal temperatures. Finally, with $1.35 \mathrm{MW}$ X2 power the suprathermal population is optically thick and central channels of the ECE radiometer measure the suprathermal temperature directly. 




(a) X2 preheating phase

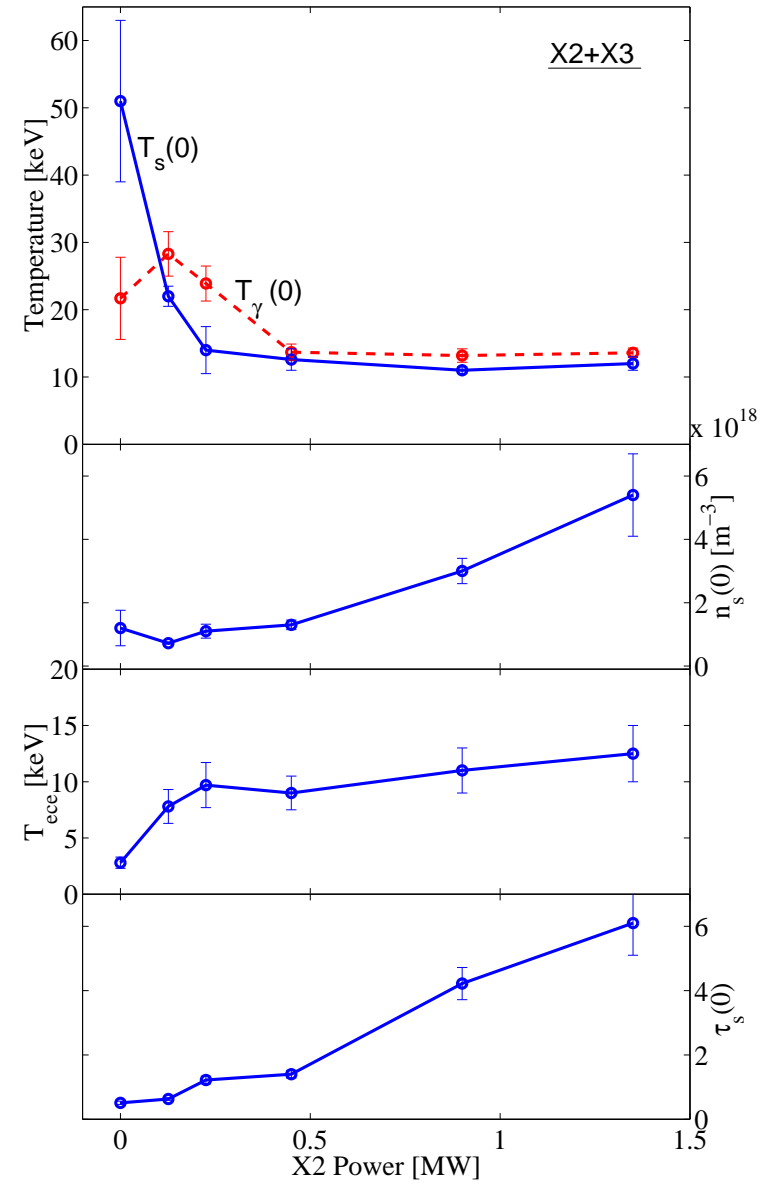

(b) $\mathrm{X} 2+\mathrm{X} 3$ phase

Figure 13. Results from the bi-Maxwellian method applied on a X2 power scan with $0.45 \mathrm{MW}$ X3 ECH power. From top to bottom: 1) Solid line represents the central suprathermal temperature $T_{s}(0)$, dashed line represents the central photon temperature $T_{\gamma}(0)$. 2) Central suprathermal density $n_{s}(0)$. 3) Central ECE temperature. 4) Second harmonic central optical depth for the suprathermals $\tau_{s}(0) \propto$ $n_{s} \cdot T_{s}$

\subsection{Estimation of the X3 absorption on the suprathermal population}

Fig. 8 shows that when X2 is launched in CO-ECCD mode with $\phi_{a b s}=+25^{\circ}$, global $\mathrm{X} 3$ absorption is measured to be $100 \%$ by the DML, even though TORAY-GA predicts only $50 \%$ absorption i.e. $\tau_{b}^{X 3}=0.7$. Applying the bi-Maxwellian method to this shot gives $T_{s}(0)=14 \pm 6 \mathrm{keV}$ and $n_{s}(0)=1.3 \cdot 10^{18} \pm 0.4 \cdot 10^{18} \mathrm{~m}^{-3}$. Calculation of the X3 beam absorption on the suprathermal population is then calculated by TORAY-GA with the suprathermal density and temperature profiles obtained and gives $\tau_{s}^{X 3}=0.5$. The first pass X3 absorption on the bulk and on the suprathermal population is given by $1-\exp \left(-\left(\tau_{b}^{X 3}+\tau_{s}^{X 3}\right)\right)=70 \%(<100 \%)$. Several effects can explain this discrepancy. Firstly, the suprathermal Maxwellian $f_{s}$ has no drift velocity with respect to the bulk 
Maxwellian. Adding such a drift in the suprathermal Maxwellian tends to increase the absorption coefficient[29]. Secondly, to consider the electron distribution function as a bi-Maxwellian is a strong approximation. An effort is currently underway to couple an ECE code to the Fokker-Planck code CQL3D in order to perform simulations of ECE from numerical distribution functions.

\section{Conclusions}

High power X2 ECCD and/or X3 ECH injection creates a suprathermal electron population whose electron cyclotron emission is measured with an ECE radiometer with antennae on the high field side of the tokamak. It has been shown that radiometry in such a configuration is very sensitive to the non-thermal EC emission. ECE on TCV has been shown to have a strong dependence on the X2 toroidal launching angle and on the X3 poloidal launching angle.

Under the assumption that the electron distribution function is a linear combination of two Maxwellians, the first one describing the bulk and the second one the suprathermal population, the resulting ECE can be described by a superposition of the bulk and suprathermal emissions. As the bulk of typical EC heated plasmas on TCV is optically thick for the second harmonic of $\Omega_{c e}$ in X-mode, ECE is determined mainly by the value of the optical depth of the suprathermal population. The bi-Maxwellian modelling applied on an X2 power scan, has shown that with X2 heating only suprathermal electron densities between $1 \cdot 10^{17}$ and $2 \cdot 10^{18} \mathrm{~m}^{-3}$ are measured; when $\mathrm{X} 3$ power is added, densities between $1.2 \cdot 10^{18}$ and $5.4 \cdot 10^{18} \mathrm{~m}^{-3}$ are found with suprathermal temperatures from 51 to $12 \mathrm{keV}$. For optically thin X3 LFS injection, it has been shown that absorption on the suprathermal electron population explains partly the discrepancy between global and first pass absorption measurements.

\section{Appendix: optical depth}

As in our plasmas the central bulk density $n_{b}(0) \approx 2.5 \cdot 10^{19} \mathrm{~m}^{-3}$ is not much less than the cutoff density $n_{\text {cut }}^{X 2}(0) \approx 4.1 \cdot 10^{19} \mathrm{~m}^{-3}$, we have to take into account finite density effects. For perpendicular propagation at frequency $\omega$ in a $1 / R$ space-dependent magnetic field, the optical depth for the harmonic $n$ in extraordinary mode corresponding to the length $L(\mathbf{s})$ of the path in the plasma is given by[8]

$$
\tau^{X n}=\int_{0}^{L(\mathbf{s})} \alpha_{n}^{X} d s
$$

where $\alpha_{n}^{X}$ is the corresponding absorption coefficient

$$
\alpha_{n}^{X}=A_{n} \alpha_{n, 0}^{X}
$$

where

$$
\alpha_{n, 0}^{X}=\frac{n^{(2 n-1)}}{2^{n} n !}\left(\frac{\omega_{p}}{\omega_{c}}\right)^{2}\left(\frac{v_{t h}}{c}\right)^{2(n-2)} \frac{\omega_{c}}{c}\left(-F_{n+3 / 2}^{\prime \prime}\left(z_{n}\right)\right)
$$


where $\omega$ is the frequency of the radiation, $\omega_{p}$ the plasma frequency, $\omega_{c}$ the cyclotron frequency, $v_{t h}=\sqrt{\left(k_{B} T / m_{e}\right)}$ the thermal velocity and $F_{n+3 / 2}^{\prime \prime}\left(z_{n}\right)$ the imaginary part of the Shkarovsky function

$$
F_{q}\left(z_{n}\right)=-i \int_{0}^{\infty} \frac{d \tau^{\prime}}{\left(1-i \tau^{\prime}\right)^{q}} e^{i z_{n} \tau^{\prime}}
$$

with

$$
z_{n}=\left(\frac{c}{v_{t h}}\right)^{2} \frac{\omega-n \omega_{c}}{\omega}
$$

Taking into account the cold refractive index

$$
N_{\perp, c}^{2}=1-\left(\frac{\omega_{p}}{\omega}\right)^{2} \frac{\omega^{2}-\omega_{p}^{2}}{\omega^{2}-\omega_{c}^{2}-\omega_{p}^{2}}
$$

$A_{n}$ can be calculated for $n=2$ and $n=3$ as follows:

Second harmonic: $\mathrm{n}=\mathbf{2}$

$$
A_{2}=N_{\perp,+}^{\prime}\left|1+a_{2}\right|^{2} b_{2}
$$

with $N_{\perp,+}^{\prime}$ the real part of

$$
N_{\perp,+}=\sqrt{\frac{-(1+b)+\left((1+b)^{2}+4 a N_{\perp, c}^{2}\right)^{1 / 2}}{2 a}}
$$

with

$$
\begin{gathered}
a=-\frac{1}{2}\left(\frac{\omega_{p}}{\omega_{c}}\right)^{2} \frac{\omega^{2}-\omega_{c}^{2}}{\omega^{2}-\omega_{c}^{2}-\omega_{p}^{2}} F_{7 / 2}\left(z_{2}\right) \\
b=-2\left(1-\frac{\omega_{p}^{2}}{\omega\left(\omega-\omega_{c}\right)}\right) a \\
a_{2}=\frac{1}{2}\left(\frac{\omega_{p}}{\omega_{c}}\right)^{2} \frac{1+3 N_{\perp,+}^{2} F_{7 / 2}\left(z_{2}\right)}{3-\left(\omega_{p} / \omega_{c}\right)^{2}\left(1+\frac{3}{2} N_{\perp,+}^{2} F_{7 / 2}\left(z_{2}\right)\right)} \\
b_{2}=\left|1+\frac{1}{2}\left(\frac{\omega_{p}}{\omega_{c}}\right)^{2}\left(1+a_{2}\right)^{2} F_{7 / 2}^{\prime}\left(z_{2}\right)\right|^{-1}
\end{gathered}
$$

Third harmonic: $\mathrm{n}=3$

$$
A_{3}=\left(N_{\perp, c}\right)^{3}\left|1+a_{3}\right|^{2} b_{3}
$$

with

$$
\begin{gathered}
a_{3}=\frac{\left(\omega_{p} / \omega_{c}\right)^{2}}{3\left(8-\left(\omega_{p} / \omega_{c}\right)^{2}\right)} \\
b_{3}=1
\end{gathered}
$$

Finally to take into account intermediate temperature effects typically up to $20 \mathrm{keV}$, a generalisation of equation (8) to cover higher temperatures is obtained by replacing

$$
\alpha_{n, 0}^{X} \rightarrow \alpha_{n, 0}^{X} \exp \left[\gamma_{n}^{X}\left(\frac{v_{t h}}{c}\right)^{2} z_{n}\right]
$$




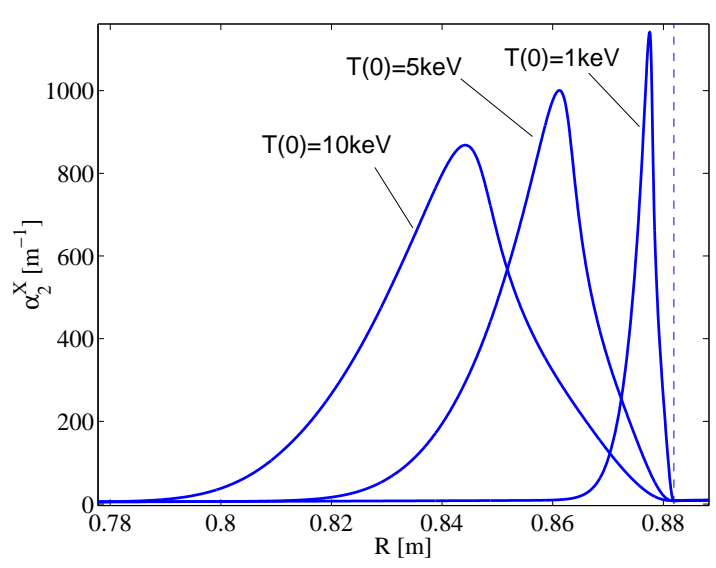

(a) $\alpha_{2}^{X}$

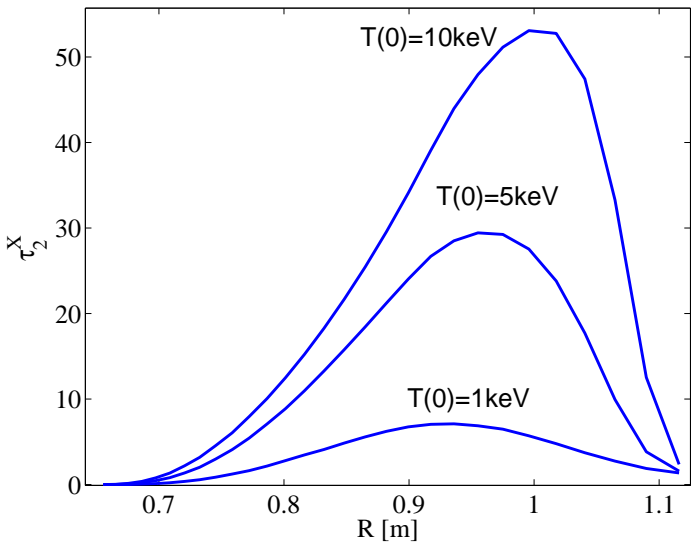

(b) $\tau^{X 2}$

Figure 14. (a): $\alpha_{2}^{X}$ calculated for $\omega=2 \cdot \Omega_{c e}(0)$ for three different central temperatures $T(0)$ with the following profiles: $T(r)=T(0) \cdot\left(1-(r / a)^{2}\right)^{3}$ and $n(r)=2 \cdot 10^{19} \cdot\left(1-(r / a)^{2}\right)^{0.5}$. The vertical dashed line represent the position of the cold resonance. (b): Radial $\tau^{X 2}$ profiles for the same parameters

where

$$
\gamma_{n}^{X}=\frac{3}{4}(2 n-3)-\frac{2 a_{n}}{1+a_{n}}+\frac{2 n^{2}}{2 n+3}\left(1+\frac{2}{n\left(1+a_{n}\right)}\right)\left(N_{\perp}^{\prime}\right)^{2}
$$

$N_{\perp}^{\prime}$ being given by equation (14) for $n=2$ and by equation (12) for $n=3$.

For the second harmonic X polarisation, Fig. 14 represents $\alpha_{2}^{X}$ and $\tau^{X 2}$ profiles for three different values of temperature. Relativistic broadening of the absorption coefficient is clearly illustrated by the displacement of the maximum of $\alpha_{2}^{X}$ to the HFS. The corresponding effect on the radial profile of $\tau^{X 2}$ is to displace its maximum to the LFS.

\section{Acknowledgements}

We wish to thank Y.Peysson and the CEA/Cadarache for the loan of the HXR camera and associated electronics to the CRPP. This work was partly supported by the Swiss National Science Foundation.

\section{References}

[1] G.Taylor et al 1992 Proceedings of the 8th Joint Workshop on ECE and ECRH, Gut Ising, Germany p. 277

[2] H.Knoepfel and D.A.Spong 1979 Nucl.Fusion 19785

[3] H.Matsuura et al 1993 Fusion Technology 2417

[4] S.Bernabei et al 1982 Phys. Rev. Lett. 491255

[5] G.Giruzzi et al 1995 Phys. Rev. Lett. 74550

[6] V.V.Alikaev and V.V.Parail 1991 Plasma Phys. Control. Fusion 331639

[7] G.E.Guest et al 1987 Nuclear Fusion 271245 
[8] M.Bornatici et al 1983 Nuclear Fusion 231153

[9] S.Alberti et al 2002 Nuclear Fusion 4242

[10] F.Engelmann and F.Curatolo 1973 Nuclear Fusion 13497

[11] H.J.Hartfuss et al 1997 Plasma Phys. Control. Fusion 391693

[12] F.Hofmann et al 1994 Plasma Phys. Control. Fusion 36 B277

[13] T.P.Goodman et al 1996 Proc. of the 19th Symposium on Fusion Technology, Lisbon, ed. C.Varandas and F.Serra (North-Holland, Amsterdam 1997) p.565

[14] Y.Peysson and F.Imbeaux 1999 Review of Scientific Instruments $\mathbf{7 0} 3987$

[15] Y.Peysson et al 2001 Nucl. Instrum. and Methods in Phys. Res.A 458269

[16] R.Behn et al 1995 Proc. 7th Int. Symp. Laser Aided Plasma Diagnostics (M.Muruora, Fukuoka, Japan)

[17] A.Manini et al 2002 Plasma Phys. Control. Fusion 44139

[18] R.Bekefi et al 1966 Radiation Processes in Plasmas John Wiley \& Sons, New-York

[19] S.Coda et al 1999 Proc. of the 26th EPS Conference on Controlled Fusion and Plasma Physics, Europhys. Conf. Abstr. 23J 1097

[20] G.R.Smith et al 1995 Proceedings of the 9th Joint Workshop on ECE and ECRH, Borrego Springs, $C A$ p. 651

[21] R.H.Cohen 1987 Phys. Fluids 302442

[22] K.Matsuda 1989 IEEE Trans. Plasma Sci. PS.17 6

[23] Y.R.Lin-Liu et al 1999 Plasma Phys. Control. Fusion 23J 1245

[24] S.Coda et al 2001 Proc. of the 28th EPS Conference on Controlled Fusion and Plasma Physics, (Madeira, Portugal), Europhys. Conf. Abstr. 25A 301

[25] R.W.Harvey et al 2002 Phys. Rev. Lett. 88205001

[26] S.Coda et al 2002 Proc. of the 29th EPS Conference on Controlled Fusion and Plasma Physics, (Montreux, Switzerland), Oral presentation 4.03

[27] M.Lontano, R.Pozzoli, E.V.Suvorov 1981 Il Nuovo Cimento 63529

[28] M.Bornatici, F.Engelmann 1994 Phys. Plasmas 1189

[29] M.Bornatici, U.Ruffina and E.Westerhof 1986 Plasma Phys. Control. Fusion 28630 